\title{
Alzheimer risk genes modulate the relationship between plasma apoE and cortical PiB binding
}

\section{OPEN}

Andreas Lazaris, BA* Kristy S. Hwang, BS* Naira Goukasian, BS Leslie M. Ramirez, BS Jennifer Eastman, MA Anna E. Blanken, BA Edmond Teng, MD, PhD Karen Gylys, RN, PhD Greg Cole, PhD Andrew J. Saykin, PsyD Leslie M. Shaw, PhD John Q. Trojanowski, $\mathrm{MD}, \mathrm{PhD}$

William J. Jagust, MD Michael W. Weiner, MD Liana G. Apostolova, MD, MSCR

For the Alzheimer's Disease Neuroimaging Initiative

Correspondence to Dr. Apostolova: lapostol@iu.edu

\section{Supplemental data} at Neurology.org/ng

\section{ABSTRACT}

Objective: We investigated the association between apoE protein plasma levels and brain amyloidosis and the effect of the top 10 Alzheimer disease (AD) risk genes on this association.

Methods: Our dataset consisted of 18 AD, 52 mild cognitive impairment, and 3 cognitively normal Alzheimer's Disease Neuroimaging Initiative 1 (ADNI1) participants with available $\left[{ }^{11} \mathrm{C}\right]$-Pittsburgh compound $\mathrm{B}(\mathrm{PiB})$ and peripheral blood protein data. We used cortical pattern matching to study associations between plasma apoE and cortical PiB binding and the effect of carrier status for the top 10 AD risk genes.

Results: Low plasma apoE was significantly associated with high PiB SUVR, except in the sensorimotor and entorhinal cortex. For BIN1 rs744373, the association was observed only in minor allele carriers. For CD2AP rs9349407 and CR1 rs3818361, the association was preserved only in minor allele noncarriers. We did not find evidence for modulation by CLU, PICALM, ABCA7, BIN1, and MS4A6A.

Conclusions: Our data show that BIN1 rs744373, CD2AP rs9349407, and CR1 rs3818361 genotypes modulate the association between apoE protein plasma levels and brain amyloidosis, implying a potential epigenetic/downstream interaction. Neurol Genet 2015;1:e22; doi: 10.1212/ NXG.0000000000000022

\section{GLOSSARY}

$\mathbf{A D}=$ Alzheimer disease $\mathbf{A D N I}=$ Alzheimer's Disease Neuroimaging Initiative; $\mathbf{A} \boldsymbol{\beta}=\boldsymbol{\beta}$-amyloid $\mathbf{C D R}=$ Clinical Dementia Rating; GWAS = genome-wide association studies; $\mathbf{M C l}=$ mild cognitive impairment; $\mathbf{M M S E}=$ Mini-Mental State Examination; $\mathbf{M V B}=$ multivesicular body; NIA = National Institute on Aging; PiB = Pittsburgh compound B; $\mathbf{S N P}=$ single nucleotide polymorphism; SUVR = standardized uptake value ratio.

The strongest genetic risk factor for late-onset Alzheimer disease $(\mathrm{AD})$ is the apolipoprotein $\mathrm{E}$ gene $(A P O E)$, with the $A P O E \varepsilon 4$ allele carrying the greatest risk via effects on $\beta$-amyloid $(\mathrm{A} \beta)$ metabolism. ${ }^{1}$ APOE codes for the apolipoprotein E protein (apoE), which plays an essential role in cholesterol metabolism, neuronal trafficking, synaptogenesis, and blood-brain barrier integrity. ${ }^{1,2}$ ApoE-containing lipoproteins bind $\mathrm{A} \beta$ and promote its clearance and degradation. ${ }^{1,2}$ The apoE4 isoform is preferentially degraded by astrocytes, leading to reduced overall brain apoE levels, reduction of $A \beta$ clearance, ${ }^{3}$ and higher $A \beta$ levels in the brain and plasma. ${ }^{1,4-6}$ Hence apoE protein plasma levels are significantly lower in patients with $\mathrm{AD}$ and in $A P O E \varepsilon 4$ carriers. $^{7}$

Amyloid PET tracers provide reliable in vivo visualization of cortical fibrillar A $\beta$ plaque deposition. The $A P O E \& 4$ genotype has been shown to positively associate with brain amyloid levels.

\footnotetext{
*Co-first authors.

From the University of California Berkeley (A.L.), Berkeley; Oakland University William Beaumont School of Medicine (K.S.H.), Rochester, MI; Department of Neurology (K.S.H., N.G., A.E.B., E.T., G.C., L.G.A.), David Geffen School of Medicine at UCLA, Los Angeles, CA; Drexel University College of Medicine (L.M.R.), Philadelphia, PA; Northwestern University Feinberg School of Medicine (J.E.), Chicago, IL; Veterans Affairs Greater Los Angeles Healthcare System (E.T., G.C.), Los Angeles, CA; School of Nursing (K.G.), UCLA, Los Angeles, CA; Department of Radiology and Imaging Sciences, Center for Neuroimaging (A.J.S., L.G.A.), Department of Neurology (L.G.A.), and Department of Medical and Molecular Genetics (L.G.A.), School of Medicine, Indiana University, Indianapolis; Department of Pathology and Laboratory Medicine (L.M.S., J.Q.T.), University of Pennsylvania School of Medicine, Philadelphia; Department of Public Health and Neuroscience (W.J.J.), UC Berkeley, CA; and Department of Veterans' Affairs Medical Center (M.W.W.), San Francisco, CA.

Coinvestigators are listed at Neurology.org/ng.

Funding information and disclosures are provided at the end of the article. Go to Neurology.org/ng for full disclosure forms. The Article Processing Charge was paid by the authors.

This is an open access article distributed under the terms of the Creative Commons Attribution-NonCommercial-NoDerivatives License 4.0 (CC BY-NC-ND), which permits downloading and sharing the work provided it is properly cited. The work cannot be changed in any way or used commercially.
} 
$A P O E \& 4$ carriers show significantly higher prevalence of Pittsburgh compound B (PiB) uptake than noncarriers across all disease stages. ${ }^{8,9}$

ApoE protein plasma levels were reported to be lower in research subjects with high PiB-PET binding in one study ${ }^{7}$ but not in another. ${ }^{10}$ Such discrepant results are difficult to reconcile and raise the question whether other genetic and perhaps even environmental factors influence this association.

Genome-wide association studies (GWAS) have identified novel $\mathrm{AD}$ risk variants. Of those, PICALM rs3851179, BIN1 rs7561528, and CR1 rs1408077 have been associated with cortical and hippocampal atrophy, ${ }^{11}$ and $A B C A 7$ rs3764650, ${ }^{12} A B C A 7$ rs3752246, ${ }^{13}$ BIN1 rs $744373,{ }^{14} C R 1$ rs6701713, ${ }^{11} C R 1$ rs3818361, ${ }^{10} C R 1$ rs6656401, and $C L U$ rs3818361 ${ }^{14}$ have been associated with PET amyloid deposition. An association between postmortem amyloid burden and CD2AP rs9349407 was reported by one group, ${ }^{12}$ but no significant relationship with $\mathrm{PiB}$ binding in nondemented elderly was observed by others. ${ }^{13}$

The exact mechanism through which these genes exert an association with amyloid burden is not clear. In this study, we assess the associations between the plasma levels of the $A P O E$ gene product - the apoE protein-and cortical $\mathrm{PiB}$ binding, and examine whether the presence of other $\mathrm{AD}$ risk variants modulates this association.

METHODS Standard protocol approvals, registrations, and patient consents. Data used in the preparation of this article were obtained from the Alzheimer's Disease Neuroimaging Initiative (ADNI) database (http://adni.loni.usc.edu). ADNI was launched in 2003 by the National Institute on Aging (NIA), the National Institute of Biomedical Imaging and Bioengineering, the US Food and Drug Administration, private pharmaceutical companies, and nonprofit organizations, as a $\$ 60$-million, 5-year, public-private partnership. The primary goal of ADNI has been to test whether serial MRI, PET, other biological markers, and clinical and neuropsychological assessment can be combined to measure clinical progression in mild cognitive impairment $(\mathrm{MCI})$ and early $\mathrm{AD}$. Determination of sensitive and specific markers of very early $\mathrm{AD}$ progression is intended to aid researchers and clinicians to develop new treatments and monitor their effectiveness, as well as lessen the time and cost of clinical trials. The principal investigator of this initiative is Michael W. Weiner, MD, VA Medical Center and University of California, San Francisco. ADNI is the result of efforts of many coinvestigators from a broad range of academic institutions and private corporations, and participants have been recruited from more than 50 sites across the United States and Canada. The initial goal of $\mathrm{ADNI}$ was to recruit 800 adults, aged 55 to 90 , to participate in the research-approximately 200 cognitively normal older individuals to be followed for 3 years, 400 people with MCI to be followed for 3 years, and 200 people with early $\mathrm{AD}$ to be followed for 2 years. For upto-date information, see www.adni-info.org.

The clinical description of the ADNI cohort has been previously published. ${ }^{15}$ The diagnosis of $\mathrm{AD}$ was based on the National Institute of Neurological and Communicative Disorders and Stroke and the $\mathrm{AD}$ and Related Disorders Association (NINCDS-ADRDA) criteria. Participants with $\mathrm{AD}$ were required to have Mini-Mental State Examination (MMSE) scores between 20 and 26 and a Clinical Dementia Rating (CDR) score of 0.5 to 1 at baseline. Qualifying MCI participants had memory complaints but no significant functional impairment, scored between 24 and 30 on the MMSE, had a global CDR score of 0.5 , a CDR memory score of 0.5 or greater, and objective memory impairment on Wechsler Memory Scale-Logical Memory II test. Healthy control participants had MMSE scores between 24 and 30 , a global CDR score of 0 , and did not meet criteria for $\mathrm{MCI}$ and AD. Participants were excluded if they refused or were unable to undergo MRI; had other neurologic disorders, active depression, or a history of psychiatric diagnosis, alcohol, or substance dependence within the past 2 years; had less than 6 years of education; or were not fluent in English or Spanish. The full list of inclusion/exclusion criteria may be accessed on pages 23-29 of the online ADNI protocol (see http://www.adni-info.org/Scientists/ADNIScientistsHome.aspx). Written informed consent was obtained from all participants. The subset of $18 \mathrm{AD}, 52 \mathrm{MCI}$, and 3 cognitively normal ADNI participants who received $\left[{ }^{11} \mathrm{C}\right]-\mathrm{PiB}$ have been included in this study.

PiB analyses. We downloaded all available PiB ADNI1 scans from the ADNI Web site (http://adni.loni.usc.edu) in October 2008. A detailed description of PiB-PET acquisition may be found at www.adni-info.org. Briefly, ADNI PiB images were collected at 12 ADNI sites. $\left[{ }^{11} \mathrm{C}\right]-\mathrm{PiB}$ with minimum $90 \%$ radiochemical purity and minimum specific activity of $300 \mathrm{Ci} /$ mmol was synthesized. Participants were injected with $15 \pm 1.5$ $\mathrm{mCi}$ PiB. Dynamic acquisition frames were obtained on a PET scanner 50-70 minutes after injection. A PiB standardized uptake value ratio (SUVR) image was obtained by averaging the individual 50-70 minutes after injection frames.

$\mathrm{PiB}$ scans were normalized to the mean $\mathrm{PiB}$ retention value of the cerebellar cortex $\left(\mathrm{SUVR}_{\mathrm{CbGM}}\right)$. After normalization, the PiB SUVR ${ }_{\text {CbGM }}$ data were spatially coregistered to the participants' baseline MRI scan using the Minctracc algorithm and 9-parameter (9P) transformation ( 3 translations, 3 rotations, 3 scales). The PiB data were smoothed with a $15-\mathrm{mm}$ kernel and convected onto the $3 \mathrm{D}$ hemispheric models derived with the cortical pattern matching technique as previously described. ${ }^{16,17}$

Genetic analyses. Genotypic data were extracted from the publicly accessible GWAS data from the ADNI Web site (http://adni. loni.usc.edu). The detailed GWAS genotyping protocol has been previously described. ${ }^{18}$ Briefly, single nucleotide polymorphism (SNP) genotyping was completed on all ADNI participants for more than 620,000 target SNPs using a total of $7 \mathrm{~mL}$ of blood from all participants. Genomic DNA was extracted using the QIAamp DNA Blood Maxi Kit (Qiagen, Inc, Valencia, CA) and analyzed using Human 610-Quad BeadChip (Illumina, Inc, San Diego, CA) according to the manufacturer's protocols (Infinium HD Assay; Super Protocol Guide; rev. A, May 2008).

$A P O E$ genotyping was carried out by PCR amplification, HhaI restriction enzyme digestion, and subsequent standard gel resolution and visualization processes. ${ }^{19,20}$ We classified participants as carriers and noncarriers for the following AD risk SNPs: BIN1 rs744373 and rs7561528; $C L U$ rs11136000, rs2279590, and rs9331888; PICALM rs3851179 and rs541458; $C R 1$ rs3818361; $A B C A 7$ rs3764650; MS4A6A rs610932; CD33 rs3826656 and rs3865444; MS4A4E rs670139; CD2AP rs9349407 and rs3865444. The following SNPs had balanced carrier/noncarrier status and were further analyzed: 
CD2AP rs9349407 (40 carriers, 33 noncarriers), $C R 1$ rs3818361 (30 carriers, 43 noncarriers), BIN1 rs744373 (42 carriers, 31 noncarriers), CD33 rs3826656 (31 carriers, 42 noncarriers), $C L U$ rs9331888 (44 carriers, 29 noncarriers), and $C L U$ rs 2279590 (47 carriers, 26 noncarriers).

Plasma biomarkers. Plasma collection was conducted according to ADNI standard operating protocols. ${ }^{21}$ Briefly, morning fasting plasma samples were obtained at each visit. The plasma samples were then sent to Rules-Based Medicine (RBM, Austin, TX) for measurement of 190 protein analytes with an immunoassay panel developed on the Luminex xMAP (Austin) platform. Each plate was run with 3 levels of quality control, and each analyte had a validation report; samples from the total ADNI plasma cohort were run on 15 plates. ApoE protein plasma levels were freely downloaded from http://adni.loni.usc.edu.

Statistical analyses. The 3D association between apoE protein plasma levels and cortical PiB SUVR $\mathrm{CbGM}_{\mathrm{C}}$ was studied using linear regression in the pooled sample using age and sex as covariates. Next, we split the pooled sample based on the presence or absence of disease- associated alleles for CD2AP rs9349407, CR1 rs3818361, BIN1 rs744373, CD33 rs3826656 and $C L U$ rs9331888 and studied the associations of the plasma apoE levels and PiB SUVR $\mathrm{CbGM}_{\text {separately }}$ in minor SNP carriers and noncarriers while adjusting for age and sex. The $3 \mathrm{D}$ associations between cortical $\mathrm{PiB} \mathrm{SUVR}_{\mathrm{CbGM}}$ and risk genotypes were studied using linear regression with age and sex as covariates. We additionally ran maps correcting for $A P O E$ genotype and diagnosis.

Our linear regression 3D statistical maps were subjected to multiple comparison correction by permutation analyses (permuting the predictor variables). Using a set-level inference approach, we defined a single corrected $p$ value for each map based on the number of points surviving a particular a priori threshold (set to 0.01 in our analyses). This approach tends to be more sensitive for detecting a distributed pattern of weak effects as opposed to the peak height of the maximum statistic, which is best for detecting a spatially highly concentrated effect.

RESULTS Demographic comparisons. All demographic variables and comparisons are shown in table 1.

Table 1 Demographic statistics of participant pool, indicated by the pooled sample and genotype

\begin{tabular}{|c|c|c|c|c|c|c|c|c|c|c|}
\hline & \multirow[b]{2}{*}{ Age, y } & \multicolumn{2}{|c|}{ Sex } & \multirow[b]{2}{*}{ Education, $y$} & \multirow[b]{2}{*}{ MMSE } & \multicolumn{3}{|c|}{ Diagnosis } & \multicolumn{2}{|l|}{ APOE $\varepsilon 4$} \\
\hline & & M & $\mathbf{F}$ & & & $\mathrm{HC}$ & $\mathrm{MCl}$ & $A D$ & Carriers & Noncarriers \\
\hline Pooled sample & $75.3 \pm 8$ & 47 & 26 & $15.9 \pm 3$ & $26.1 \pm 3$ & 3 & 53 & 17 & 42 & 31 \\
\hline \multicolumn{11}{|l|}{ BIN1 rs744373 } \\
\hline Carriers & $74.7 \pm 7$ & 29 & 13 & $15.5 \pm 3$ & $25.7 \pm 3$ & 2 & 28 & 12 & 23 & 19 \\
\hline Noncarriers & $76.2 \pm 9$ & 18 & 13 & $16.5 \pm 3$ & $26.5 \pm 3$ & 1 & 25 & 5 & 19 & 12 \\
\hline$\chi^{2}$ (p value) & 0.479 & \multicolumn{2}{|c|}{0.333} & 0.457 & \multicolumn{2}{|l|}{$0.005^{\mathrm{a}}$} & \multicolumn{2}{|l|}{0.413} & \multicolumn{2}{|c|}{0.577} \\
\hline \multicolumn{11}{|c|}{ CD2AP rs9349407 } \\
\hline Carriers & $75.7 \pm 8$ & 22 & 18 & $15.8 \pm 3$ & $25.3 \pm 3$ & 1 & 25 & 14 & 25 & 15 \\
\hline Noncarriers & $74.9 \pm 8$ & 25 & 8 & $15.9 \pm 3$ & $27.0 \pm 2$ & 2 & 28 & 3 & 17 & 16 \\
\hline$\chi^{2}$ ( $p$ value) & 0.478 & \multicolumn{2}{|c|}{0.065} & 0.858 & \multicolumn{2}{|l|}{$0.047^{a}$} & \multicolumn{2}{|l|}{$0.03^{a}$} & \multicolumn{2}{|c|}{0.345} \\
\hline \multicolumn{11}{|c|}{ CD33 rs3826656 } \\
\hline Carriers & $75.5 \pm 7$ & 17 & 14 & $15.9 \pm 3$ & $25.8 \pm 2$ & 2 & 22 & 7 & 20 & 11 \\
\hline Noncarriers & $75.2 \pm 9$ & 30 & 12 & $15.9 \pm 3$ & $26.3 \pm 3$ & 17 & 31 & 10 & 22 & 20 \\
\hline$\chi^{2}$ ( $p$ value) & 0.412 & \multicolumn{2}{|c|}{0.143} & 0.904 & \multicolumn{2}{|l|}{0.159} & \multicolumn{2}{|l|}{0.687} & \multicolumn{2}{|c|}{0.3} \\
\hline \multicolumn{11}{|c|}{ CLU rs2279590 } \\
\hline Carriers & $26.1 \pm 3$ & 29 & 18 & $15.8 \pm 3$ & $26.1 \pm 3$ & 2 & 34 & 11 & 30 & 17 \\
\hline Noncarriers & $76.0 \pm 7$ & 18 & 8 & $16.0 \pm 3$ & $26.0 \pm 3$ & 1 & 19 & 6 & 12 & 14 \\
\hline$\chi^{2}$ ( $p$ value) & 0.484 & \multicolumn{2}{|c|}{0.52} & 0.209 & \multicolumn{2}{|l|}{0.967} & \multicolumn{2}{|l|}{0.996} & \multicolumn{2}{|c|}{0.143} \\
\hline \multicolumn{11}{|l|}{ CLU rs9331888 } \\
\hline Carriers & $77.2 \pm 8$ & 29 & 15 & $15.9 \pm 3$ & $26.2 \pm 3$ & 0 & 25 & 5 & 23 & 21 \\
\hline Noncarriers & $72.4 \pm 7$ & 18 & 11 & $15.8 \pm 3$ & $25.9 \pm 4$ & 3 & 28 & 12 & 19 & 10 \\
\hline$x^{2}$ (p value) & 0.412 & \multicolumn{2}{|c|}{0.737} & 0.315 & \multicolumn{2}{|l|}{0.531} & \multicolumn{2}{|l|}{0.145} & 0.2 & \\
\hline CR1 rs381836 & & & & & & & & & & \\
\hline Carriers & $76.4 \pm 8$ & 19 & 11 & $16.4 \pm 3$ & $25.9 \pm 3$ & 1 & 34 & 9 & 16 & 14 \\
\hline Noncarriers & $74.6 \pm 8$ & 28 & 15 & $15.5 \pm 3$ & $26.1 \pm 3$ & 2 & 19 & 8 & 26 & 17 \\
\hline$\chi^{2}$ (p value) & 0.48 & 0.8 & & 0.507 & 0.92 & & 0.444 & & 0.5 & \\
\hline
\end{tabular}

Abbreviations: $\mathrm{AD}=$ Alzheimer disease; $\mathrm{HC}=$ healthy control; $\mathrm{MCl}=$ mild cognitive impairment; $\mathrm{MMSE}=$ Mini-Mental State Examination.

Age, sex, and MMSE displayed in mean \pm SD.

${ }^{a}$ Two-tailed $\chi^{2}$ test $p$ value with significant differences. Only genes that had balanced distribution between carriers and noncarriers were considered. 
Figure 1 Significance maps displaying the associations between cortical Pittsburgh compound B (PiB) binding and plasma apoE protein in the pooled sample (top panel) and stratified by the BIN1, CD2AP, and CR1 risk genotypes (bottom 3 panels)
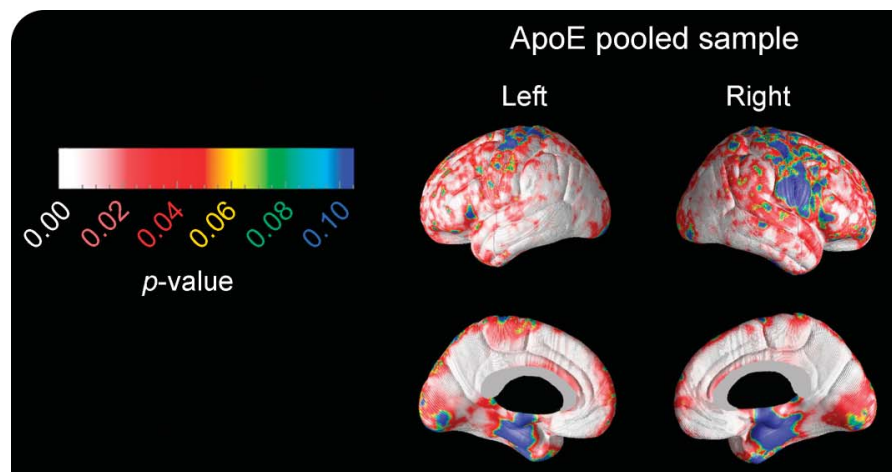

Left Right

Left

Right

BIN1 rs744373 carriers

BIN1 rs744373 noncarriers
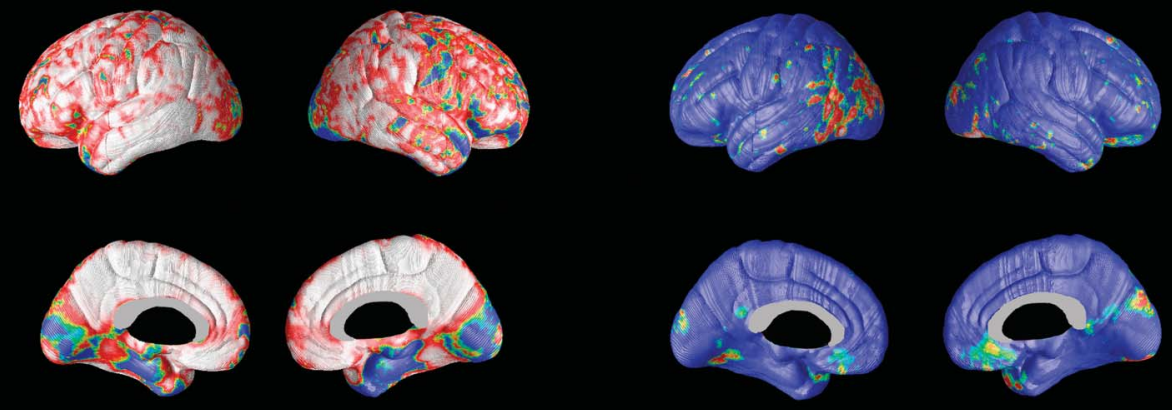

CD2AP rs9349407 carriers

CD2AP rs9349407 noncarriers
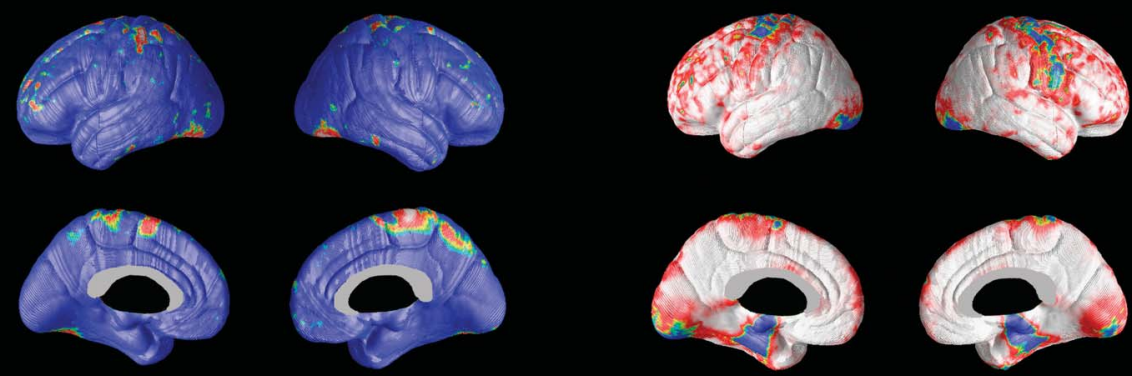

CR1 rs 3818361 carriers

CR1 rs 3818361 noncarriers
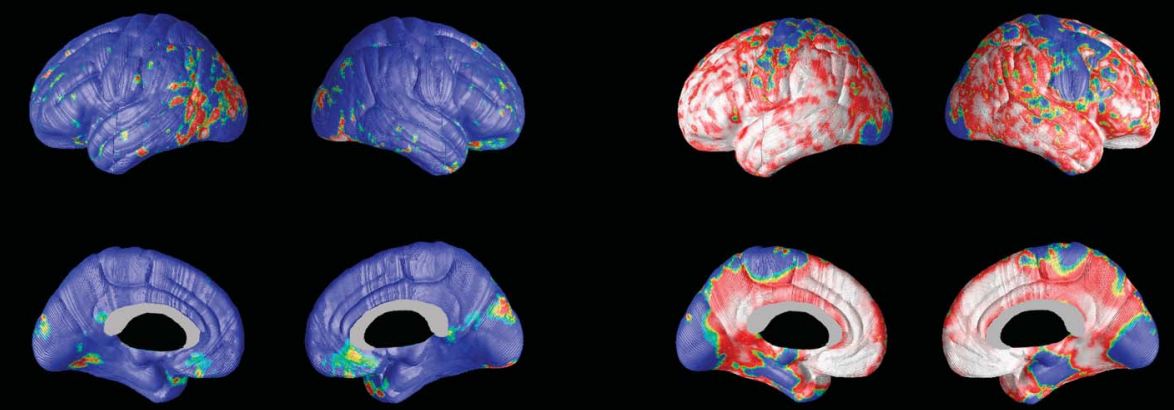

We found no significant differences in age, sex distribution, or level of education between carriers and noncarriers for each gene. There were significantly more $\mathrm{MCI}$ than $\mathrm{AD}$ and healthy control subjects among
$C D 2 A P$ noncarriers $(p=0.03)$. BIN1 and CD2AP carriers had lower MMSE scores than noncarriers (BIN1 mean MMSE 25.7 vs 26.5, $p=0.005$; CD2AP mean MMSE 25.3 vs $27.0 p=0.047$ ). Of note, there 
were no significant differences in $A P O E$ \&4 status between carriers and noncarriers for any risk gene.

None of the $\mathrm{AD}$ risk genotypes showed significant association with PiB SUVR ${ }_{\mathrm{CbGM}}$ in the pooled sample. Plasma apoE levels showed an association with $\mathrm{PiB}$ SUVR throughout the brain with the exception of the sensorimotor and entorhinal cortex (figure 1) across the pooled sample (left $p_{\text {corr }}=0.004$, right $p_{\text {corr }}=0.008$ ).

Our analyses showed no evidence of modulation of the apoE protein-PiB association by $C L U$ rs9331888 and CLU rs2279590. Plasma apoE levels showed an association with $\mathrm{PiB}$ SUVR $\mathrm{CbGM}_{\text {only in }}$ $C D 2 A P$ rs9349407 and CR1 rs3818361 minor allele noncarriers (CD2AP rs 9349407 noncarriers left $p_{\text {corr }}=$ 0.003 , right $p_{\text {corr }}=0.004 ; C R 1$ rs3818361 noncarriers left $p_{\text {corr }}=0.008$, right $p_{\text {corr }}=0.01$, figure 1 and table 2). These results remained unchanged after correcting for diagnosis (CD2AP rs9349407 noncarriers left $p_{\text {corr }}=0.009$, right $p_{\text {corr }}=0.008 ; C R 1$ rs3818361 noncarriers left $p_{\text {corr }}=0.014$, right $p_{\text {corr }}=$ 0.025 , table 2 and figure e- 1 at Neurology.org/ng). After correcting for $A P O E \& 4$, the association between apoE protein plasma levels and $\mathrm{PiB}$ binding remained for $C D 2 A P$ (left $p_{\text {corr }}=0.03$, right $p_{\text {corr }}=0.03$ ) but not CR1 (table 2 and figure e-1). Plasma apoE showed an association with $\mathrm{PiB}$ SUVR $_{\mathrm{CbGM}}$ in BIN1 rs744373 minor allele carriers (left $p_{\text {corr }}=0.006$, right $p_{\text {corr }}=0.01$, figure 1 and table 2). This BIN1 association remained after correcting for diagnosis (left $p_{\text {corr }}=0.007$, right $p_{\text {corr }}=0.017$, figure e- 1 ) and $A P O E \& 4$ genotype (left $p_{\text {corr }}=0.028$, right $p_{\text {corr }}=$ 0.038 , table 2 and figure e- 1 ).

DISCUSSION Our data show that BIN1 rs744373, $C D 2 A P$ rs9349407, and CR1 rs3818361 genotypes modulate the association between apoE protein plasma levels and brain amyloidosis. In the pooled sample, we saw the expected association between plasma apoE levels and PiB SUVR throughout the brain. Yet, when stratified by $\mathrm{AD}$ risk genotypes, plasma apoE showed a significant association with

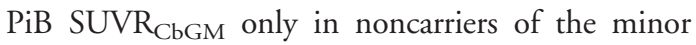
allele of CD2AP rs9349407 and CR1 rs3818361 and only in carriers of the BIN1 rs744373 minor allele. These findings imply a downstream interaction between these genes and the apoE disease-associated pathways and suggest a direct or modulatory role on $\mathrm{A} \beta$ accumulation and clearance. Brain and plasma apoE4 levels are believed to be lower than apoE3 levels because of a difference in their fate after they are endocytosed and routed to multivesicular bodies that function as sorting endosomes. ApoE3 is packaged into recycling endosomes and retroendocytosed back to the surface and secreted, whereas apoE4 is typically trafficked on through to the lysosome and degraded. Plasma apoE levels reflect this difference in processing between apoE3 and apoE4. 22,23

Bridging integrator 1 , or $B I N 1$, encodes a nucleocytoplasmic adaptor protein abundantly expressed in the CNS that is involved in synaptic vesicle endocytosis, immune response, calcium homeostasis, and apoptosis. ${ }^{24}$ The protein encoded by BIN1 has been shown to link the microtubule cytoskeleton and cellular membrane ${ }^{25}$ and has been implicated in amyloid precursor protein turnover and hence $A \beta$ production through clathrin-mediated amyloid precursor protein endocytosis. ${ }^{24,26}$ BIN1 rs744373 minor allele carriers have been reported to have an increased risk of $\mathrm{AD} .^{27}$ Increased BIN1 rs744373 expression is associated with later disease onset and shorter disease duration. ${ }^{24}$ Postmortem, no association with amyloid plaque burden

\begin{tabular}{|c|c|c|c|c|c|c|}
\hline \multirow[t]{4}{*}{ Table 2} & $\begin{array}{l}\text { ficant } p \text { values } \\
N 1, C D 2 A P, \text { ar }\end{array}$ & $\begin{array}{l}\text { the associati } \\
\text { CR1 carrier } \mathrm{s}\end{array}$ & $\begin{array}{l}\text { between pla } \\
\text { us }\end{array}$ & apoE levels & cortical PiB & ding stratified \\
\hline & \multicolumn{6}{|l|}{ Covariates } \\
\hline & \multicolumn{2}{|l|}{ Age/sex } & \multicolumn{2}{|c|}{ Age/sex/diagnosis } & \multicolumn{2}{|c|}{ Age/sex/APOE $\_4$} \\
\hline & L hemisphere & $\mathrm{R}$ hemisphere & L hemisphere & $\mathrm{R}$ hemisphere & L hemisphere & $\mathrm{R}$ hemisphere \\
\hline \multicolumn{7}{|l|}{ BIN1 } \\
\hline Carriers & $0.006^{a}$ & $0.01^{a}$ & $0.007^{a}$ & $0.017^{a}$ & $0.028^{a}$ & $0.038^{a}$ \\
\hline Noncarriers & NS & NS & NS & NS & NS & NS \\
\hline \multicolumn{7}{|l|}{$C D 2 A P$} \\
\hline Carriers & NS & NS & NS & NS & NS & NS \\
\hline Noncarriers & $0.003^{a}$ & $0.004^{\mathrm{a}}$ & $0.009^{a}$ & $0.008^{a}$ & $0.03^{a}$ & $0.03^{a}$ \\
\hline \multicolumn{7}{|l|}{ CR1 } \\
\hline Carriers & NS & NS & NS & NS & NS & NS \\
\hline Noncarriers & $0.008^{a}$ & $0.01^{a}$ & $0.014^{a}$ & $0.025^{a}$ & NS & NS \\
\hline
\end{tabular}

Abbreviations: NS = not significant; $\mathrm{PiB}=$ Pittsburgh compound $\mathrm{B}$.

a Significant. 
has been observed for BIN1 rs7561528, ${ }^{12}$ and BIN1 rs $744373,{ }^{24}$ consistent with our results in the pooled sample. An association of BIN1 rs744373 minor allele with in vivo amyloid deposition measured with ${ }^{18} \mathrm{~F}$-florbetapir has been reported, ${ }^{14}$ but this effect disappeared in the presence of the protective PICALM minor allele at rs3851179. BIN1 rs744373 also failed to show an association with CSF A $\beta$ levels. ${ }^{28}$ In our study, the presence of the BIN1 rs744373 risk variant was associated with preserved and potentially enhanced association between apoE protein plasma levels and PiB binding, lending evidence for a potential downstream functional interaction between the $B I N 1$ and $A P O E$ genes. BIN1 rs 744373 increases mRNA expression and protein expression. ${ }^{29}$ As mentioned above, apoE levels are strongly related to the classic clathrin-dependent receptor-mediated endocytosis with impaired recycling of apoE4 isoforms. ${ }^{22}$ BIN1 is an integral part of the sorting nexin-4/BAR complexes. These complexes have been shown to regulate the Rab5/7 switch that influences the transition from early to late endosomes and controls traffic to the lysosomes. They also seem to regulate Rab4/Rab11 transition to recycling endosomes. ${ }^{30} \mathrm{~A} \beta$ and apoE are known for trafficking through Rab5/Rab7 to lysosomes and to Rab11 recycling endosomes for degradation. This occurs in an apoE isoform-dependent manner in which apoE3 promotes greater $\mathrm{A} \beta$ and apoE traffic to the lysosome for clearance. ${ }^{31}$ Therefore, BIN1 is ideally positioned to regulate both $A \beta$ and apoE influx into lysosomes and trafficking toward degradation or recycling through retroendocytosis. Thus higher BIN1 expression can be hypothesized to increase apoE recycling and elevate protein levels notably, and this would be linked to faster amyloid clearance and less deposition associated with higher apoE levels.

$C D 2 A P$, the CD2-associated protein gene, codes for a scaffolding protein that plays a role in the formation of tight junctions, endocytosis, cellular waste management, and immune response. ${ }^{24} C D 2 A P$ has an established role in endocytosis, vesicle trafficking within the cell, and formation of the cytoskeleton. ${ }^{32}$ $C D 2 A P$ is involved in endosome trafficking, ${ }^{33}$ binds directly to Rab4, and regulates recycling endosomes, ${ }^{34}$ which play an essential role in the retroendocytosis of apoE that controls apoE levels. ${ }^{22}$ Consistent with this, $C D 2 A P$-knockout mice have deficits in multivesicular body (MVB) formation and endosomal-lysomal trafficking. ${ }^{29}$ Intraneuronal $A \beta$ accumulation occurs in MVBs, thereby impairing their function. ${ }^{35}$ This supports a possible interaction between $C D 2 A P$ and $A \beta$ metabolism. CD2AP rs9349407 has been associated with postmortem plaque burden and $\mathrm{AD}$ status. ${ }^{36,37}$ However, studies have failed to find a direct association between this SNP and PiB binding in ADNI. ${ }^{13}$ Our results further elaborate on this complex relationship.
We found that the association between apoE protein plasma levels and $\mathrm{PiB}$ binding is no longer present in minor allele carriers, indicating that $C D 2 A P$ influences amyloid pathology. Since CD2AP rs9349407 trends toward reducing CD2AP expression in human brain, ${ }^{29}$ one might hypothesize that decreased CD2AP (or altered interaction with binding partners) could reduce apoE's isoform-dependent recycling and endosomal-lysosomal traffic that regulates both apoE levels and $\mathrm{A} \beta$ clearance in lysosomes. ${ }^{31}$ The latter has been implicated as a cause for both low plasma apoE4 and defects in $A \beta$ clearance. ${ }^{31}$ Furthermore, endocytosed apoE isoforms differentially recycle and degrade in lysosomes. ${ }^{22}$ They also regulate endosomal-lysosomal A $\beta$ clearance. ${ }^{31}$ Since CD2AP rs9349407 trends toward reducing CD2AP expression in human brain, ${ }^{29}$ one might hypothesize that decreased CD2AP (or altered interaction with binding partners) could reduce apoE's isoform-dependent recycling and endosomal-lysosomal traffic that regulates both apoE levels and $\mathrm{A} \beta$ clearance in lysosomes. ${ }^{31}$ Thus, lowering CD2AP could diminish the correlations between $\mathrm{PiB}$ and plasma apoE.

The CR1 gene encodes complement component $3 \mathrm{~b} / 4 \mathrm{~b}$ receptor 1 - a membrane glycoprotein found on erythrocytes, leukocytes, glomerular podocytes, and splenic follicular dendritic cells. This protein mediates cell binding and removal of complement-activated immune complexes. ${ }^{24}$ CR1 serves to mediate clearance of immune complexes and phagocytosis by neutrophils and monocytes ${ }^{38}$ and plays a role in antigen presentation to B lymphocytes. ${ }^{39}$ Increased CR1 expression has been associated with $\mathrm{AD}$ diagnosis and worse cognition. $^{24}$ The presence of the CR1 rs3818361 risk variant is associated with lower $\mathrm{PiB}$ binding in 2 separate cognitively normal cohorts including an ADN1 subsample ${ }^{10}$ but not in the full ADNI1 sample. ${ }^{14}$ At the same time, 2 other SNPs, CR1 rs6656401 and rs6701713, showed an association with increased amyloid plaque burden postmortem. ${ }^{12,40}$ In this study we observed that CR1 rs3818361 minor allele carrier status leads to a loss of the association between apoE protein plasma levels and $\mathrm{PiB}$ binding, which disappeared in the presence of $A P O E \& 4$ as covariate. An epistatic interaction between the APOE genotype and CR1 rs3818361 has been previously reported. ${ }^{41,42}$

Several strengths and limitations of this study should be acknowledged. ADNI is the premier longitudinal biomarker study in AD. ADNI uses unified subject assessment, MRI, PiB-PET, CSF, and peripheral blood collection protocols and meticulous data quality control across all study sites. One of the major limitations of our study is its small sample size limited by $\mathrm{PiB}$ availability in ADNI1. Significant disproportion between carriers and noncarriers for the remaining ADNI risk SNPs led to the exclusion of these variants, 
allowing us to test our hypothesis for only 6 SNPs across $5 \mathrm{AD}$ risk genes. Furthermore, other unknown genetic interactions may also affect the plasma apoEcortical PiB associations. Last but not least, ADNI uses rigorous exclusion criteria typical of clinical trials and the study population is not representative of the general population, which may negatively affect the generalizability of our results.

The ADNI PET core chose cerebellar gray matter as the reference region for intensity normalization of ADNI1 PiB data. This is one of the commonly used approaches. Other regions that have been used in the amyloid PET literature include whole cerebellum, periventricular white matter, whole brainstem, and composite regions derived from more than one of these measures. Recently, a group of PET experts compared the performance of 4 of these normalization regions: whole cerebellum, cerebellar gray matter, pons, and whole cerebellum plus brainstem. The authors concluded that normalization to the pons performed worse than normalization strategies including the cerebellum. The 2 regions that performed best (i.e., had lowest SUVR variance) were whole cerebellum and whole cerebellum plus brainstem. ${ }^{43}$ Our study uses the cerebellar gray matter, which performed better than pons but resulted in more noise (higher SUVR variance) in the normalized data. Yet despite this potentially suboptimal signal-to-noise ratio, we were able to find significant associations.

Overall, our findings imply an interaction between several $\mathrm{AD}$ risk gene minor alleles and $A P O E$ genotype-driven brain amyloidosis. In the absence of a direct association with brain amyloidosis, we found that several $\mathrm{AD}$ risk genes nonetheless exert a modulatory effect on one of the most fundamental disease-associated pathophysiologic events-APOE $\varepsilon 4$-facilitated brain amyloid deposition. These findings lend the basis for further exploration of the exact AD-related pathophysiologic mechanisms of these genes and their products, which might ultimately lead to new therapeutic strategies.

\section{AUTHOR CONTRIBUTIONS}

Andreas Lazaris is a co-first author of the manuscript, responsible for study design, data processing, statistical analyses, and drafting of the manuscript. Kristy S. Hwang is a co-first author of the manuscript, assisting with study design, data processing, and the completion of integral statistical analyses. Ms. Hwang also authored several segments of the manuscript and took part in all revisions. Naira Goukasian completed some of the final analysis of our data and took part in revising of the manuscript. Leslie M. Ramirez completed some of the initial analyses of our data and took part in revising of the manuscript. Jennifer Eastman completed some of the initial analyses of our data and took part in revising of the manuscript. Anna E. Blanken completed some of the final analyses of our data and took part in revising of the manuscript. Edmond Teng took part in some analysis and interpretations of the data and participated in revising of the manuscript. Karen Gylys provided critical insights for interpretation of our results and participated in revising of the manuscript. Greg Cole provided critical insights for interpretation of our results and participated in revising of the manuscript. Andrew Saykin contributed to the overall ADNI study design, data collection, and genetic analyses. He also provided critical insights for interpretation of our results and participated in revising of the manuscript. Leslie Shaw contributed to the overall ADNI study design, data collection, and proteomic analyses. $\mathrm{He}$ also provided critical insights for interpretation of our results and participated in revising of the manuscript. John Q. Trojanowski contributed to the overall ADNI study design, data collection, and proteomic analyses. He also provided critical insights for interpretation of our results and participated in revising of the manuscript. William J. Jagust contributed to the overall ADNI study design, data collection, and amyloid PET data analyses. He also provided critical insights for interpretation of our results and participated in revising of the manuscript. Michael W. Weiner is the principal investigator of ADNI. He has contributed to the overall ADNI study design, data collection, and data analyses. He provided critical insights for interpretation of our results and participated in revising of the manuscript. Liana G. Apostolova is the senior author of this manuscript and is responsible for the study concept and design. She provided major oversight over all analyses, interpretation of results, and participated in writing of the manuscript.

\section{ACKNOWLEDGMENT}

Data used in the preparation of this article were obtained from the Alzheimer's Disease Neuroimaging Initiative (ADNI) database (http://adni. loni.usc.edu). As such, the investigators within the ADNI contributed to the design and implementation of ADNI and/or provided data but only some participated in analysis or writing of this report. ADNI investigators include (complete listing available at http://adni.loni.usc.edu/wp-content/ uploads/how_to_apply/ADNI_Acknowledgement_List.pdf). The authors thank the members of the ADNI Imaging Core for their contributions to the image preprocessing, the members of the ADNI Biomarker Core for the CSF biomarker analyses and the investigators at the University of Pittsburgh for the PiB SUVR analyses.

\section{STUDY FUNDING}

Data collection and sharing for this project was funded by the Alzheimer's Disease Neuroimaging Initiative (ADNI) (NIH grant U01 AG024904) and DOD ADNI (Department of Defense award W81XWH-12-2-0012). ADNI is funded by the National Institute on Aging, the National Institute of Biomedical Imaging and Bioengineering, and through generous contributions from the following: Alzheimer's Association; Alzheimer's Drug Discovery Foundation; BioClinica, Inc; Biogen Idec, Inc; BristolMyers Squibb Company; Eisai, Inc; Elan Pharmaceuticals, Inc; Eli Lilly and Company; F. Hoffmann-La Roche Ltd and its affiliated company Genentech, Inc; GE Healthcare; Innogenetics, N.V.; IXICO Ltd; Janssen Alzheimer Immunotherapy Research \& Development, LLC; Johnson \& Johnson Pharmaceutical Research \& Development LLC; Medpace, Inc; Merck \& Co, Inc; Meso Scale Diagnostics, LLC; NeuroRx Research; Novartis Pharmaceuticals Corporation; Pfizer, Inc; Piramal Imaging; Servier; Synarc, Inc; and Takeda Pharmaceutical Company. The Canadian Institutes of Health Research is providing funds to support ADNI clinical sites in Canada. Private sector contributions are facilitated by the Foundation for the NIH (www.fnih.org). The grantee organization is the Northern California Institute for Research and Education, and the study is coordinated by the Alzheimer's Disease Cooperative Study at the University of California, San Diego. ADNI data are disseminated by the Laboratory for Neuro Imaging at the University of Southern California. The CSF biomarker and PiB SUVR analyses reported in this manuscript were funded by the Easton Consortium for Alzheimer's Drug Discovery and Biomarker Development, NIA R01 AG040770, NIA K02 AG048240, and NIA P50 AG16570.

\section{DISCLOSURE}

Andreas Lazaris, Kristy S. Hwang, Naria Goukasian, Leslie M. Ramirez, Jennifer Eastman, and Anna E. Blanken report no disclosures. Edmond Teng owns stock in General Electric and Cerner Corporations; has served on the editorial board for Dementia and Geriatric Cognitive Disorders; and has received research support from Eli Lilly, Biogen, Genentech, Merck, and K08 AG 34628 (jointly sponsored by NIA, AFAR, the John A. Hartford Foundation, the Atlantic Philanthropies, the Starr Foundation, and an anonymous donor). Karen Gylys has served on the editorial 
board for Acta Neuropahtologica Communications; and has received research support from NIH R01 AG027465-01A2, P50 AG16570, UCLA Alzheimer's Disease Research Center, and the Lincy Foundation. Greg Cole holds patents for an FDDNP PET probe for protein aggregates, Curcumin formulation for enhanced bioavailability with lipidation and antioxidant stabilization, and Medical Food for brain health, provides unpaid advice to Neurovision Imaging, which is using his and coholders' patent pending curcumin formulation for retinal imaging; has received research support from NIH NCCIH AT006816, Greater LA VA, and GRECC; receives royalties for the following: FDDNP UCLA patent royalties, Curcumin formulation/UCLA/VA patent royalties, and Medical Food-UCLA/VA patent royalties; and was a an expert witness for legal proceedings regarding WhiteWave (makers of Horizon Milk). Andrew Saykin serves as ADNI Genetics Core Leader; receives research support from Siemens Medical Solutions and Welch Allyn, and from the following Alzheimer's disease related NIH grants: R01 AG19771, R01 CA101318, R01 LM011360, U01 AG032984, RC2 AG036535, and P30 AG10133; has served as a consultant to Siemens Healthcare, Eli Lilly, and Arkley BioTek; has served on the advisory board for Siemens Healthcare and Eli Lilly; has received honoraria from Siemens Healthcare; and has served on the editorial board of Brain Imaging and Behavior (a Springer Journal). Leslie Shaw has been a consultant to Innogenetics and collaborates on quality assessment activities as part of the Alzheimer's Disease Neuroimaging Initiative; has been a consultant for Janssen and Novartis; serves as consultant, member of advisory board and has received speaker fees and travel expenses from Eli Lilly and Company; has served on the editorial board for Therapeutic Drug Monitoring; and has received research support from Eisai, NIH, and the Alzheimer's Disease Neuroimaging Initiative. John Q. Trojanowski has served on the editorial board of Alzheimer's $\&$ Dementia; may accrue revenue on patents submitted by the University of Pennsylvania wherein he is inventor including: modified avidin-biotin technique; method of stabilizing microtubules to treat Alzheimer's disease; method of detecting abnormally phosphorylated tau; method of screening for Alzheimer's disease or disease associated with the accumulation of paired helical filaments; compositions and methods for producing and using homogeneous neuronal cell transplants; rat comprising straight filaments in its brain; compositions and methods for producing and using homogeneous neuronal cell transplants to treat neurodegenerative disorders and brain and spinal cord injuries; diagnostic methods for Alzheimer's disease by detection of multiple MRNAs; methods and compositions for determining lipid peroxidation levels in oxidant stress syndromes and diseases; compositions and methods for producing and using homogenous neuronal cell transplants; method of identifying, diagnosing, and treating alpha-synuclein positive neurodegenerative disorders; mutation-specific functional impairments in distinct tau isoforms of hereditary frontotemporal dementia and parkinsonism linked to chromosome-17: genotype predicts phenotype; microtubule stabilizing therapies for neurodegenerative disorders; and treatment of Alzheimer's and related diseases with an antibody; is coinventor on patents submitted the University of Pennsylvania wherein he is inventor that have generated income he has received from the sale of Avid to Eli Lily including: amyloid plaque aggregation inhibitors and diagnostic imaging agents; and has received research support from the Marian S. Ware Alzheimer Program and Benaroya. William J. Jagust has served as a consultant to Banner Alzheimer Institute, Genentech, Inc, Synarc, Janssen Alzheimer Immunotherapy, F. Hoffman LA Roche, Novartis, and Siemens; has served on the scientific advisory boards of Genentech, Inc and Novartis; has served on the editorial boards of Frontiers in Human Neuroscience, Annals of Neurology, Brain Imaging and Behavior, Alzheimer's Disease and Associated Disorders, and Neuroimage: Clinical; and has received research support from Avid Radiopharmaceuticals F-AV-45A14, NIH grants AG034570, AG025303, AG044292, AG012435, AG021028, AG031563, AG019724, AG030048, AG032306, and AG024904, and the Tau Consortium (Rainwater Foundation). Michael W. Weiner has served on the Scientific Advisory Boards for Pfizer, BOLT International, Neurotrope Bioscience, Alzheon, University of Pennsylvania's Neuroscience of Behavior Initiative, National Brain Research Center (NBRC), India, LEARN Program at University of North Carolina, Dolby Family Ventures, LP, ADNI, and Eli Lilly; has provided consulting to Synarc, Pfizer, Janssen, KLJ Associates, Easton Associates, Harvard University, University of California, Los Angeles (UCLA), Alzheimer's Drug Discovery Foundation (ADDF), Neurotrope Bioscience, Avid
Radiopharmaceuticals, ClearView Healthcare Partners, Perceptive Informatics, Smartfish AS, Decision Resources, Inc, Araclon, Merck, Defined Health, Howard University, Bogen Idec, BoClinica, and Genentech; has received travel funding from Pfizer, Paul Sabatier University, MCI Group France, Travel eDreams, Inc, Neuroscience School of Advanced Studies (NSAS), Danone Trading, BV, CTAD Ant Congres, Kenes, Intl, ADRC, UCLA, UCSD, ADCS, Sanofi-Aventis Groupe, University Center Hospital, Toulouse, Araclon, AC Immune, Eli Lilly, New York Academy of Sciences (NYAS), National Brain Research Center, Northwestern University, Fidelity Biosciences Research Initiative, University of Pennsylvania, The Alzheimer's Association, Merck, ADPD, Alzheimer's Drug Discovery Foundation (ADDF), Tokyo University, Kyoto University, Cornell-Weill University, Rockafeller University, Memorial Sloan-Kettering Cancer Center, Biogen, and India for Johns Hopkins Medicine; has received honoraria from Pfizer, Tohoku University, Consortium for Multiple Sclerosis Centers (CMSC), and Danone Trading, $\mathrm{BV}$; has received research support from Merck and Avid; has been an employee of the University of California, San Francisco and the San Francisco VA Medical Center; has received research support from Merck, Avid, Lilly, Alzheimer's Diseases Discovery Foundation (ADDF), the Veterans Administration (VA), Department of Defense, from the following grants: 2U01AG024904, W81XWH-13-1-0259, W81XWH-12-2-0012, R01 AG10897, 1P41 EB015904, P01 AG19724, R01 AG032306, R01A G03879, ADNI 2-12-233036, 20110506, R01 MH098062-01, and from NIH/NIA/National Institute of Mental Health, DOD, Alzheimer's Association, Alzheimer's Drug Discovery Foundation, Merck, Avid, and the Veterans Administration (VA). Liana G. Apostolova has served as a consultant to Lilly and GE Healthcare; has received speaker honoraria from Eli Lilly; has served on the editorial boards of Alzheimer and Dementia: Diagnosis, Assessment and Disease Monitoring; has served on the speakers bureaus of Eli Lilly and GE Healthcare; and has received research support from GE Healthcare and NIA. Go to Neurology.org/ng for full disclosure forms.

Received June 21, 2015. Accepted in final form August 13, 2015.

\section{REFERENCES}

1. Kim J, Basak JM, Holtzman DM. The role of apolipoprotein E in Alzheimer's disease. Neuron 2009;63: 287-303.

2. Liu CC, Kanekiyo T, Xu H, Bu G. Apolipoprotein E and Alzheimer disease: risk, mechanisms and therapy. Nat Rev Neurol 2013;9:106-118.

3. Riddell DR, Zhou H, Atchison K, et al. Impact of apolipoprotein E (ApoE) polymorphism on brain ApoE levels. J Neurosci 2008;28:11445-11453.

4. Morris JC, Roe CM, Xiong C, et al. APOE predicts amyloid-beta but not tau Alzheimer pathology in cognitively normal aging. Ann Neurol 2010;67:122-131.

5. Bales KR, Du Y, Holtzman D, Cordell B, Paul SM. Neuroinflammation and Alzheimer's disease: critical roles for cytokine/Abeta-induced glial activation, NF-kappaB, and apolipoprotein E. Neurobiol Aging 2000;21:427-432; discussion 451-423.

6. Rebeck GW, Reiter JS, Strickland DK, Hyman BT. Apolipoprotein $\mathrm{E}$ in sporadic Alzheimer's disease: allelic variation and receptor interactions. Neuron 1993;11:575-580.

7. Gupta VB, Laws SM, Villemagne VL, et al. Plasma apolipoprotein $\mathrm{E}$ and Alzheimer disease risk: the AIBL study of aging. Neurology 2011;76:1091-1098.

8. Fleisher AS, Chen $\mathrm{K}$, Liu X, et al. Using positron emission tomography and florbetapir F18 to image cortical amyloid in patients with mild cognitive impairment or dementia due to Alzheimer disease. Arch Neurol 2011;68:1404-1411.

9. Reiman EM, Chen K, Liu X, et al. Fibrillar amyloid-beta burden in cognitively normal people at 3 levels of genetic risk for Alzheimer's disease. Proc Natl Acad Sci USA 2009; 106:6820-6825. 
10. Thambisetty M, An Y, Nalls M, et al. Effect of complement CR1 on brain amyloid burden during aging and its modification by APOE genotype. Biol Psychiatry 2013; 73:422-428.

11. Braskie MN, Ringman JM, Thompson PM. Neuroimaging measures as endophenotypes in Alzheimer's disease. Int J Alzheimers Dis 2011;2011:490140.

12. Shulman JM, Chen K, Keenan BT, et al. Genetic susceptibility for Alzheimer disease neuritic plaque pathology. JAMA Neurol 2013;70:1150-1157.

13. Hughes TM, Lopez OL, Evans RW, et al. Markers of cholesterol transport are associated with amyloid deposition in the brain. Neurobiol Aging 2014;35: 802-807.

14. Hohman TJ, Koran ME, Thornton-Wells T; Alzheimer's Neuroimaging Initiative. Epistatic genetic effects among Alzheimer's candidate genes. PLoS One 2013;8:e80839.

15. Petersen RC, Aisen PS, Beckett LA, et al. Alzheimer's Disease Neuroimaging Initiative (ADNI): clinical characterization. Neurology 2010;74:201-209.

16. Apostolova LG, Hwang KS, Medina LD, et al. Cortical and hippocampal atrophy in patients with autosomal dominant familial Alzheimer's disease. Dement Geriatr Cogn Disord 2011;32:118-125.

17. Thompson PM, Hayashi KM, de Zubicaray G, et al. Dynamics of gray matter loss in Alzheimer's disease. J Neurosci 2003;23:994-1005.

18. Saykin AJ, Shen L, Foroud TM, et al. Alzheimer's Disease Neuroimaging Initiative biomarkers as quantitative phenotypes: genetics core aims, progress, and plans. Alzheimers Dement 2010;6:265-273.

19. Hixson JE, Vernier DT. Restriction isotyping of human apolipoprotein E by gene amplification and cleavage with HhaI. J Lipid Res 1990;31:545-548.

20. Reymer PW, Groenemeyer BE, van de Burg R, Kastelein JJ. Apolipoprotein E genotyping on agarose gels. Clin Chem 1995;41:1046-1047.

21. Arnold SE, Xie SX, Leung YY, et al. Plasma biomarkers of depressive symptoms in older adults. Transl Psychiatry 2012;2:e65.

22. Heeren J, Beisiegel U, Grewal T. Apolipoprotein E recycling: implications for dyslipidemia and atherosclerosis. Arterioscler Thromb Vasc Biol 2006;26: 442-448.

23. Rellin L, Heeren J, Beisiegel U. Recycling of apolipoprotein $\mathrm{E}$ is not associated with cholesterol efflux in neuronal cells. Biochim Biophys Acta 2008;1781: 232-238.

24. Karch CM, Goate AM. Alzheimer's disease risk genes and mechanisms of disease pathogenesis. Biol Psychiatry 2015; 77:43-51.

25. Meunier B, Quaranta M, Daviet L, Hatzoglou A, Leprince C. The membrane-tubulating potential of amphiphysin 2/BIN1 is dependent on the microtubulebinding cytoplasmic linker protein 170 (CLIP-170). Eur J Cell Biol 2009;88:91-102.

26. Tan MS, Yu JT, Tan L. Bridging integrator 1 (BIN1): form, function, and Alzheimer's disease. Trends Mol Med 2013;19:594-603.
27. Schellenberg GD, Montine TJ. The genetics and neuropathology of Alzheimer's disease. Acta Neuropathol 2012; 124:305-323.

28. Kauwe JS, Cruchaga C, Karch CM, et al. Fine mapping of genetic variants in BIN1, CLU, CR1 and PICALM for association with cerebrospinal fluid biomarkers for Alzheimer's disease. PLoS One 2011;6:e15918.

29. Karch CM, Jeng AT, Nowotny P, Cady J, Cruchaga C, Goate AM. Expression of novel Alzheimer's disease risk genes in control and Alzheimer's disease brains. PLoS One 2012;7:e50976.

30. van Weering JR, Verkade P, Cullen PJ. SNX-BAR-mediated endosome tubulation is co-ordinated with endosome maturation. Traffic 2012;13:94-107.

31. Li J, Kanekiyo T, Shinohara M, et al. Differential regulation of amyloid- $\beta$ endocytic trafficking and lysosomal degradation by apolipoprotein E isoforms. J Biol Chem 2012; 287:44593-44601.

32. Lynch DK, Winata SC, Lyons RJ, et al. A Cortactin-CD2associated protein (CD2AP) complex provides a novel link between epidermal growth factor receptor endocytosis and the actin cytoskeleton. J Biol Chem 2003;278:21805-21813.

33. Kim JM, Wu H, Green G, et al. CD2-associated protein haploinsufficiency is linked to glomerular disease susceptibility. Science 2003;300:1298-1300.

34. Cormont M, Metón I, Mari M, et al. CD2AP/CMS regulates endosome morphology and traffic to the degradative pathway through its interaction with Rab4 and c-Cbl. Traffic 2003;4:97-112.

35. Almeida CG, Takahashi RH, Gouras GK. Beta-amyloid accumulation impairs multivesicular body sorting by inhibiting the ubiquitin-proteasome system. J Neurosci 2006;26:4277-4288.

36. Naj AC, Jun G, Beecham GW, et al. Common variants at MS4A4/MS4A6E, CD2AP, CD33 and EPHA1 are associated with late-onset Alzheimer's disease. Nat Genet 2011;43: 436-441.

37. Hollingworth P, Harold D, Sims R, et al. Common variants at ABCA7, MS4A6A/MS4A4E, EPHA1, CD33 and CD2AP are associated with Alzheimer's disease. Nat Genet 2011;43:429-435.

38. Krych-Goldberg M, Moulds JM, Atkinson JP. Human complement receptor type 1 (CR1) binds to a major malarial adhesin. Trends Mol Med 2002;8:531-537.

39. Dunkelberger JR, Song WC. Complement and its role in innate and adaptive immune responses. Cell Res 2010;20: $34-50$.

40. Chibnik LB, Shulman JM, Leurgans SE, et al. CR1 is associated with amyloid plaque burden and age-related cognitive decline. Ann Neurol 2011;69:560-569.

41. Carrasquillo MM, Belbin O, Hunter TA, et al. Replication of BIN1 association with Alzheimer's disease and evaluation of genetic interactions. J Alzheimers Dis 2011;24:751-758.

42. Omoumi A, Fok A, Greenwood T, Sadovnick AD, Feldman HH, Hsiung GYR. Evaluation of late-onset Alzheimer disease genetic susceptibility risks in a Canadian population. Neurobiol Aging 2014;35:936. e935-e912.

43. Klunk WE, Koeppe RA, Price JC, et al. The Centiloid Project: standardizing quantitative amyloid plaque estimation by PET. Alzheimers Dement 2015;11:1-15. e11-e14. 


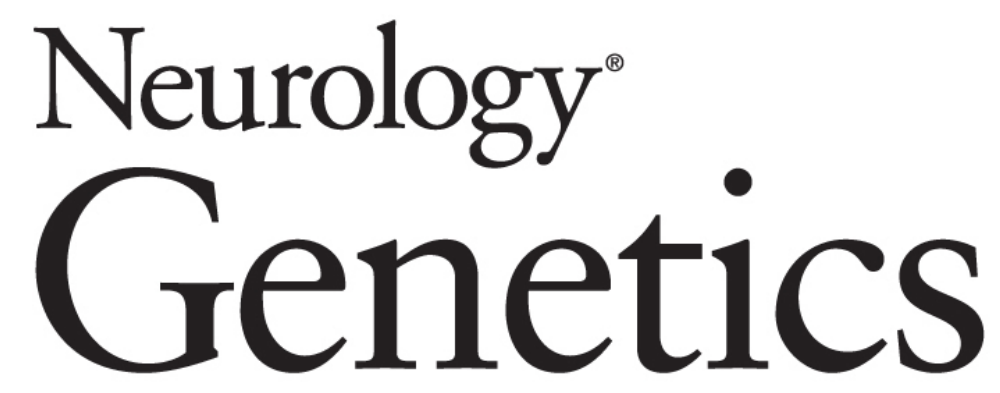

Alzheimer risk genes modulate the relationship between plasma apoE and cortical PiB binding

Andreas Lazaris, Kristy S. Hwang, Naira Goukasian, et al.

Neurol Genet 2015;

DOI 10.1212/NXG.0000000000000022

This information is current as of October 15, 2015

Neurol Genet is an official journal of the American Academy of Neurology. Published since April 2015, it is an open-access, online-only, continuous publication journal. Copyright $(2015$ American Academy of Neurology. All rights reserved. Online ISSN: 2376-7839.

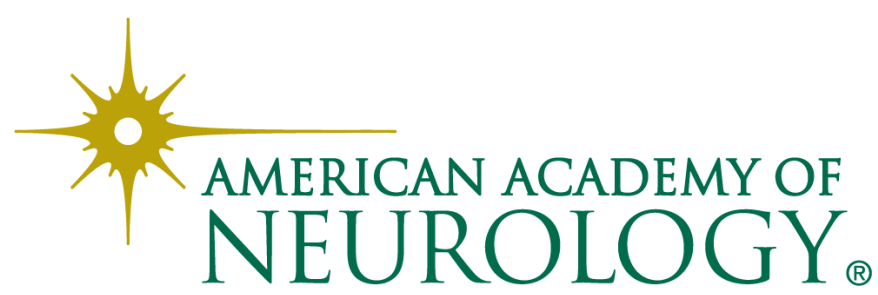




\section{Updated Information \& Services}

\section{Supplementary Material}

\section{References}

Citations

Subspecialty Collections

\section{Permissions \& Licensing}

\section{Reprints}

including high resolution figures, can be found at: http://ng.neurology.org/content/1/3/e22.full.html

Supplementary material can be found at: http://ng.neurology.org/content/suppl/2015/10/19/1.3.e22.DC1 http://ng.neurology.org/content/suppl/2015/10/19/1.3.e22.DC2

This article cites 43 articles, 10 of which you can access for free at: http://ng.neurology.org/content/1/3/e22.full.html\#\#ref-list-1

This article has been cited by 1 HighWire-hosted articles: http://ng.neurology.org/content/1/3/e22.full.html\#\#otherarticles

This article, along with others on similar topics, appears in the following collection(s):

Alzheimer's disease

http://ng.neurology.org//cgi/collection/alzheimers_disease

Association studies in genetics

http://ng.neurology.org//cgi/collection/association_studies_in_genetics

\section{CT}

http://ng.neurology.org//cgi/collection/ct

MCI (mild cognitive impairment)

http://ng.neurology.org//cgi/collection/mci_mild_cognitive_impairmen $\mathrm{t}$

\section{PET}

http://ng.neurology.org//cgi/collection/pet

Information about reproducing this article in parts (figures,tables) or in its entirety can be found online at:

http://ng.neurology.org/misc/about.xhtml\#permissions

Information about ordering reprints can be found online: http://ng.neurology.org/misc/addir.xhtml\#reprintsus

Neurol Genet is an official journal of the American Academy of Neurology. Published since April 2015, it is an open-access, online-only, continuous publication journal. Copyright $(2015$ American Academy of Neurology. All rights reserved. Online ISSN: 2376-7839.

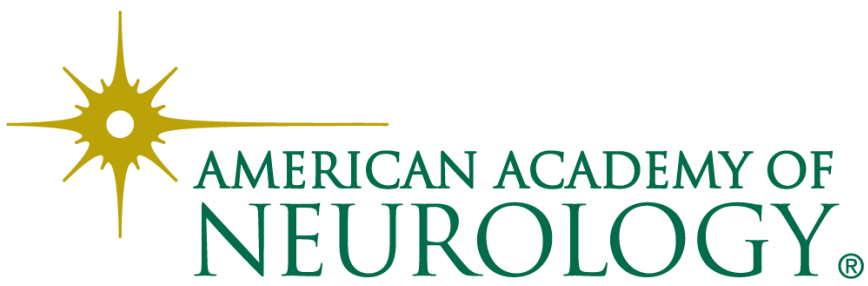

